\title{
Álgebra más allá de letras y números: Oportunidades para desarrollar el pensamiento algebraico en la Educación Primaria
}

Álgebra além de letras e números: Oportunidades para desenvolver o pensamento algébrico na educação básica

Beyond letters and numbers: Developing Algebraic thinking in the Elementary School

Eder Pinto

Facultad de Educación, Universidad del Desarrollo (UDD)

Santiago, Chile

E-mail: epinto@udd.cl

Orcid: 0000-0003-1911-4158

Cristina Ayala-Altamirano

Facultad de Ciencias de la Educación, Universidad de Almería (UAL)

Almería, España

E-mail: altamira@ual.es

Orcid: 0000-0002-9165-9470 
Resumen: En este artículo presentamos ideas que podrían ayudar a profesores y profesoras a fomentar el pensamiento algebraico de sus estudiantes de Educación Primaria (6-12 años). $\mathrm{Si}$ bien diferentes países incluyen la idea de álgebra (y pensamiento algebraico) en sus normativas curriculares, todavía faltan elementos que ayuden a ver el álgebra más allá de letras y números. Los objetivos de este artículo son: (a) caracterizar elementos conceptuales y teóricos involucrados en el pensamiento algebraico, y (b) reflexionar qué decisiones pedagógicas podrían fomentar el pensamiento algebraico de estudiantes de Educación Primaria. Para abordar ambos objetivos, presentamos las respuestas de algunos estudiantes que participaron en diferentes sesiones de un taller de matemáticas, durante el verano de 2021. En concreto, presentamos tres problemas que trabajaron los estudiantes y exponemos algunas de sus respuestas (orales y escritas). A partir de dichas respuestas, resaltamos oportunidades para desarrollar el pensamiento algebraico en los diferentes cursos de la Educación Primaria.

Palabras claves: pensamiento algebraico, álgebra escolar, Educación Primaria.

\begin{abstract}
:
Neste artigo, apresentamos idéias que poderiam ajudar os professores a fomentar o pensamento algébrico em seus alunos da escola primária (6-12 anos de idade). Embora países diferentes incluam a idéia de álgebra (e pensamento algébrico) em suas regulamentações curriculares, ainda há falta de elementos que ajudem a ver a álgebra além de letras e números. Os objetivos deste artigo são: (a) caracterizar elementos conceituais e teóricos envolvidos no pensamento algébrico, e (b) refletir sobre quais decisões pedagógicas poderiam fomentar o pensamento algébrico nos alunos do ensino fundamental. Para abordar ambos os objetivos, apresentamos as respostas de alguns alunos do ensino fundamental que participaram de diferentes sessões de uma oficina de matemática durante o verão de 2021. Especificamente, apresentamos três problemas nos quais os estudantes trabalharam e apresentamos algumas de suas respostas (orais e escritas). A partir destas respostas, destacamos as oportunidades para desenvolver o pensamento algébrico nas diferentes séries do Ensino Fundamental.
\end{abstract}

Keywords: pensamento algébrico, álgebra escolar, educação primária.

Resumen: In this article, we present ideas for fostering algebraic thinking in elementary school students (6-12 years old). Although different countries include the idea of algebra (and algebraic thinking) in their curricula guidelines, elements that help to see algebra beyond letters and numbers are still missing. The objectives of this article are: (a) to characterize conceptual and theoretical elements involved in algebraic thinking, and (b) to reflect on what pedagogical decisions could promote algebraic thinking in the elementary school. To address both objectives, we present the responses of some elementary school students who participated in different sessions of a mathematics Summer School. Specifically, we present three problems that the students worked on and present some of their responses (oral and written). Based on these responses, we highlight opportunities to develop algebraic thinking in the different grades of elementary school Education.

Key words: Algebraic thinking, school algebra, elementary school.

Recebido em 08/08/2021

Aceito em 18/10/2021 


\section{INTRODUCCIÓN}

Actualmente, diversos países incluyen en sus normativas curriculares la idea de que el pensamiento algebraico debe fomentarse desde la Educación Primaria (e incluso Infantil). La incorporación de este tipo de pensamiento matemático surge como una respuesta al esfuerzo de diversos actores de la comunidad científica que, desde finales de los años 80 , han ido mostrando evidencias de cómo niños y niñas perciben y organizan regularidades, trabajan con estructuras y son capaces de generalizar; aspectos centrales del pensamiento algebraico. A pesar de los esfuerzos anteriores, todavía existen desafíos sobre cómo promover este tipo de pensamiento y entregar herramientas concretas a profesores y profesoras para que identifiquen las oportunidades de desarrollar el pensamiento algebraico en sus aulas.

A través de este artículo buscamos mostrar algunos caminos efectivos que podrían ayudar a profesores y profesoras a fomentar el pensamiento algebraico de sus estudiantes. En concreto, los siguientes objetivos dirigen este trabajo:

- Caracterizar elementos conceptuales y teóricos involucrados en el pensamiento algebraico.

- Reflexionar qué decisiones pedagógicas podrían fomentar el pensamiento algebraico de estudiantes de Educación Primaria.

Comenzamos este artículo con una aproximación teórica a algunas de las ideas relacionadas con el pensamiento algebraico en los primeros cursos y que, a lo largo de nuestras investigaciones, nos ha ayudado a dar sentido a las respuestas de niñas y niños de Educación Primaria. Luego, presentamos las respuestas de algunos estudiantes de cuarto de primaria (9-10 años) que participaron en diferentes sesiones de un taller de matemáticas. Esas sesiones tenían por propósito guiar a los estudiantes en la expresión y justificación de ideas matemática generales, a través de diferentes contenidos del álgebra escolar. Finalmente, presentamos algunas reflexiones que podrían inspirar la toma de decisiones de docentes de Educación Primaria, con la finalidad de que sus estudiantes atiendan a las propiedades y regularidades matemáticas, más que a cálculos aritméticos aislados. 


\section{Pensamiento algebraico en Educación Primaria}

Pareciera existir un acuerdo en la comunidad científica al señalar que el pensamiento algebraico no se trata solo de símbolos; más bien involucra formas de pensar (Kieran, 2011). Sin embargo, lograr una definición única sobre este tipo de pensamiento es reconocido como una tarea compleja. En este artículo adoptamos la idea que el pensamiento algebraico es aquel que se refiere a cantidades indeterminadas (incógnitas, variables, parámetros o números generalizados) y trata dichas cantidades de forma analítica, es decir, aunque las cantidades sean desconocidas, se suman, restan, multiplican o dividen, como si fueran conocidas (Radford, 2018). Al pensar algebraicamente se razona sobre la generalidad, se nota una estructura algebraica y se estudian los cambios (Kieran, 2004).

A partir de las ideas de Kaput (2008), el pensamiento algebraico se organiza en tres líneas de contenido: (a) aritmética generalizada, en la cual las operaciones aritméticas son usadas como un contexto para desarrollar pensamiento algebraico, focalizándose en sus propiedades fundamentales; (b) equivalencia, expresiones, ecuaciones e inecuaciones, la cual incluyen una comprensión relacional del signo igual y favorece que los estudiantes vean las expresiones como objetos en vez de ver estas como una serie de cálculos aislados; y (c) pensamiento funcional, la cual se centra en la relación entre cantidades que covarían. A su vez, se incluyen prácticas del pensamiento algebraico que son transversales a dichos contenidos: generalización, representación, justificación y razonamiento acerca de la generalidad.

La generalización constituye el elemento central del pensamiento algebraico. Se busca que los y las estudiantes atiendan a lo que está ocurriendo siempre, en cualquier caso. Las maneras en que estudiantes expresan ideas matemáticas generales incluyen representaciones tan variadas como el lenguaje natural, gestos, dibujos, tablas, gráficos, números y símbolos. Por tanto, el pensamiento algebraico puede tomar lugar antes que se introduzca la notación algebraica (Radford, 2018). 


\section{PRODUCCIONES ORALES Y ESCRITAS DE ESTUDIANTES}

Durante inicios del año 2021 trabajamos con un grupo de 21 niños y niñas de cuarto de primaria (9-10 años). Los estudiantes participaron, de manera online, en diez sesiones de un taller de matemáticas que buscaba guiarlos en la expresión y justificación de ideas matemáticas generales, al trabajar con diferentes contenidos del álgebra escolar. Organizamos las sesiones en torno a las líneas de contenido de pensamiento algebraico antes mencionadas.

Las respuestas que presentamos aquí emergen en la última sesión con los estudiantes, en el contexto de grupos de trabajo pequeños. Presentamos tres problemas a los estudiantes, quienes disponían del problema impreso y contaban con pizarras y marcadores para expresar sus ideas, si así lo deseaban. Específicamente, un profesor se reúne con 4-5 niñas y niños, y cada estudiante responde de manera individual a las preguntas que se plantean, para luego compartir sus respuestas con el grupo en una puesta en común. Nuestro interés era identificar la comprensión que mostraban las y los estudiantes a las tareas propuestas luego de la intervención, e identificar las estrategias que utilizaban para resolverlas.

A continuación, presentamos los tres problemas presentados y algunas respuestas que consideramos representativas de este grupo de estudiantes.

\section{Sentencias Numéricas: ¿Verdadero o falso?}

El primer problema que presentamos a los estudiantes atiende a la aritmética generalizada como línea de contenido al pensamiento algebraico. En la Figura 1 presentamos el problema que resolvieron los y las estudiantes. 
Explica si cada relación es Verdadera o Falsa (V/F)
a) $39+121=121+39$
b) $28+7+7=26+14$
c) $12+3=10+5$
d) $57+22=58+21$

Figura 1. Sentencias numéricas

Fuente: Adaptado de Blanton et al. (2019).

El objetivo del problema presentado (ver Figura 1) es establecer relaciones de igualdad y tratar de responder sin realizar ningún tipo de cálculo. Presentamos cuatro sentencias numéricas, donde cada una involucra alguna propiedad de la operatoria. Las sentencias "a", "c" y "d" son verdaderas. Mientras la sentencia "a" se relaciona con la propiedad conmutativa de la adición, las sentencias "c" y "d" con la compensación. La sentencia "b" es falsa y se relaciona con la propiedad asociativa de la adición. Nuestro interés estaba puesto en identificar si los estudiantes recurren a cálculos aritméticos para indicar la veracidad o falsedad de las sentencias, o bien, si solo atienden a la estructura de las sentencias y desde ahí establecer conclusiones.

Sobre las respuestas de los estudiantes, dos hechos nos llamaron profundamente la atención. En primer lugar, gran parte de los estudiantes no realizó cálculos aritméticos para indicar si las sentencias eran verdaderas o falsas. Para muchos de ellos les bastó con "mirar" la sentencia y descubrir la respuesta. En segundo lugar, algunos estudiantes emplearon vocabulario matemático y propiedades matemáticas para responder a las preguntas. Para ilustrar lo anterior, en el siguiente extracto presentamos las ideas de Julia $(\mathrm{J})$ e Ignacio (I) al responder a la primera sentencia $(39+121=121+39)$.

1. Profesor $(P)$ : La sentencia "a", ¿verdadera o falsa?

2. J: Verdadera. Son los mismos sumandos, solo que cambian de posición, están invertidos.

3. P: $\quad$ Y tú, Ignacio. ¿Qué opinas?

4. I: $\quad$ Es verdadero, porque es conmutativo, solo se invierten los números.

5. P: ¿Qué es conmutativo, Ignacio? 


\section{Universidade Federal da Grande Dourados}

6. D:

Es eso, que simplemente están invertidos los números, no hay ningún número diferente.

Para lograr que las y los estudiantes respondieran del modo que antes se describe, en el diseño de las clases fue fundamental la justificación. En un inicio, como es de esperar, las y los estudiantes recurrieron a conocimientos cercanos para ellos, por ejemplo, realizar cálculos o emplear lenguaje matemáticamente impreciso o coloquial. En el curso de las discusiones, los docentes relacionaron las ideas de los estudiantes y los motivaron a buscar caminos alternativos en los que no fuera necesario el cálculo, también los motivaron a emplear lenguaje matemático preciso, reconociendo que sus respuestas eran correctas, pero también se podrían expresar de otros modos. Estas prácticas fueron efectivas, tal como se muestra en el extracto, aparece la idea de sumandos (línea 2) para referirse a los números que están a cada lado del signo igual, y la propiedad conmutativa de la adición (línea 4).

La actividad de interpretar sentencias numéricas podría parecer una simple actividad de cálculo y respuesta única, sin embargo, desde una visión algebraica de la aritmética, este tipo de actividad es una oportunidad de interpretar los números como ejemplos y otorgarles un carácter general. Para fomentar la generalización, se sugiere motivar a los estudiantes a buscar estrategias para responder sin calcular o relacionar las sentencias con otros ejemplos numéricos en los que suceda los mismo, luego preguntar porque siempre las sentencias son verdaderas. En el caso de las sentencias falsas, se podría preguntar qué cambios se podrían realizar para que la sentencia sea verdadera. En ambos casos los estudiantes pueden proponer múltiples respuestas válidas, por lo que es una buena oportunidad para discutir e introducir el vocabulario matemático, por ejemplo, hablando explícitamente de las propiedades de las operaciones. En el trabajo realizado por Molina y Castro (2021) se muestran otros ejemplos de tareas en las que se reflexiona en torno a diversas propiedades aritméticas con estudiantes de primaria. 


\section{LA CAJA DE GALLETAS}

El segundo problema que presentamos a los y las estudiantes aparece en la Figura 2. Este problema atiende a la equivalencia, expresiones, ecuaciones e inecuaciones como línea de contenido al pensamiento algebraico.

Juan y Sandra tienen cada uno una caja con galletas. Saben que sus cajas tienen la misma cantidad de galletas, pero no saben cuántas. Sandra también tiene 3 galletas en su mano.

a) ¿Cómo representarías el número de galletas que tiene Juan?

b) ¿Cómo representarías el número de galletas de Sandra?

c) ¿Qué relación hay entre el número de galletas de Juan y Sandra?

d) Supongamos que Sandra cuenta todas sus galletas y descubre que tiene 14 en total. ¿Cuántas galletas tenía en la caja?

e) ¿Podrías saber cuántas galletas tiene Juan?

Figura 2. Problema de la caja de galletas

Fuente: Adaptación de Carraher y Schliemann (2007)

El objetivo del problema es doble, por un lado, se busca que las y los estudiantes expresen ideas matemáticas que involucran cantidades indeterminadas (preguntas "a", "b" y "c") y por otro, resolver ecuaciones que involucran una incógnita (preguntas "d" y "e"). El siguiente extracto muestra la respuesta de Luis (L) a la primera pregunta (¿Cómo representarías el número de galletas que tiene Juan?).

7. Profesor $(\mathrm{P})$ : ¿Cómo representaste el número de galletas que tiene Juan, Luis?

8. L: Bueno, no sabemos cuántas [galletas] tiene. Sabemos que Sandra tiene tres en su mano. Sabemos que tienen la misma cantidad. Pero no podemos representar cuál es esa cantidad.

9. P: ¿Por qué no podemos representar esa cantidad?

10.L: Bueno, podemos representarlo, pero con letras. Tengo $x$ galletas, y eso.

11.P: ¿Por qué pensaste en letras?

12. L Porque podríamos representarlo de cualquier forma.

Para responder problemas como el que se presenta en la Figura 2 los estudiantes tienen que aceptar el álgebra como un lenguaje para expresar situaciones en las que hay cantidades indeterminadas involucradas. En las ideas es Luis es 


\section{Universidade Federal da Grande Dourados}

posible observar que reconoce que la situación involucra una cantidad indeterminada (línea 8) y al interpretar sus argumentos inferimos que señala que la cantidad de galletas no se puede representar con un número en concreto, pero sí se puede representar a través de letras.

En este caso, para que los estudiantes lograran expresar diversas situaciones con notación algebraica, en el diseño de las sesiones se les motivó a contar historias matemáticas sin resolver cálculos. En primera instancia, por medio de la discusión se les motivó a reconocer los elementos importantes de cada enunciado y relacionarlo con expresiones matemáticas, es decir reconocer la estructura subyacente de la situación. Si los estudiantes no han resuelto situaciones que involucran cantidades indeterminadas, en primera instancia se sugiere contar historias matemáticas con sentencias numéricas, sin calcular y luego modificar aquellas historias o proponer otras que involucran cantidades indeterminadas.

Según lo reportado en diversas investigaciones el uso de la letra requiere de negociación de significados, por lo que antes de su uso se pueden emplear otras representaciones, ya sea lenguaje natural o símbolos que los estudiantes sugieran. En Ayala-Altamirano y Molina (2021) se describe una experiencia en la que los estudiantes representan cantidades indeterminadas empleando la palabra "muchos" cuando expresan relaciones generales.

\section{EL PROBLEMA DEL TREN}

El último problema que presentamos a los estudiantes atiende al pensamiento funcional como línea de contenido al pensamiento algebraico (ver Figura 3). El problema involucra la función $y=2 x+1$ y buscaba encontrar una relación entre un número de paradas (variable independiente) y el correspondiente número de personas que va en un tren (variable dependiente). 
Susana conduce un tren que recorre diferentes ciudades de nuestro país. En cada ciudad que para el tren, 2 personas suben.

a) Luego de salir de la 1ำ parada, ¿cuántas personas van el tren? ¿Cómo lo sabes?

b) Luego de salir de la 4ํㅜ parada, ¿cuántas personas van el tren? ¿Cómo lo sabes?

c) Luego de salir de la $2^{\circ}$ parada, ¿cuántas personas van el tren? ¿Cómo lo sabes?

d) Luego de salir de la $7^{\circ}$ parada, ¿cuántas personas van el tren? ¿Cómo lo sabes?

e) Luego de salir de la 100ํaa. ¿Cuántas personas van el tren? ¿Cómo lo sabes?

f) A partir de tus respuestas anteriores, ¿cómo puedes saber cuántas personas van luego de cualquier parada? ¿Cómo lo sabes?

Figura 3. Problema del tren

Fuente: Adaptado de Pinto y Cañadas (2018)

El problema del tren proporcionó un espacio productivo para descubrir regularidades, relacionar cantidades y descubrir, por ejemplo, cuántas personas van en el tren luego de cualquier número de paradas. Nuestro interés era identificar si las reglas (funcionales) que construían los estudiantes al responder a las preguntas "a""d" eran extendidas para responder a una cantidad "lejana" (100) e indeterminada (cualquiera) de número de paradas.

Al responder a las preguntas que involucraban un caso particular $(a-e)$, la mayoría de los estudiantes estableció una regla que relacionaba la cantidad de personas y el número de parada correspondiente. Estas reglas involucraban diferentes operaciones aritméticas y en el siguiente extracto presentamos la respuesta que dio un estudiante, Teo $(\mathrm{T})$, al responder a las primeras preguntas.

13. Profesor $(P)$ : Teo, luego de salir de la primera parada, ¿cuántas personas van en el tren?

14.T:

Tres.

15.P: ¿Por qué? ¿Cómo lo sabes?

16. T: (...) Multipliqué el uno por dos y le sumé 1, que es la conductora.

17.P: $\quad$ Y luego de salir de la cuarta parada. ¿Cuántas personas van en el tren?

18.T: $\quad$ Multipliqué cuatro por dos, y me dio ocho, y más la conductora, nueve.

19. ¿Y luego de salir de la segunda parada?

20. Multipliqué el dos por dos y me dio cuatro. Luego, le sumé uno y me dio cinco. 


\section{Universidade Federal da Grande Dourados}

Teo encontró una regla que involucraba multiplicar por dos el número de paradas, para luego sumar uno, que corresponde a la conductora. Él ha notado que algo se mantiene constante a través de los diferentes números de parada: multiplicar por dos y sumar uno. Al parecer, Teo encontró una regla general que aplicó a todos los casos preguntados.

En la respuesta a la última pregunta (¿cómo puedes saber cuántas personas van luego de cualquier parada? ¿Cómo lo sabes?), la Figura 5 presenta la respuesta escrita de dos estudiantes: Julia (en "a") y Cristóbal (en "b"). A diferencia de Teo, ellos emplearon la letra para representar cualquier cantidad y expresar de modo general la relación funcional.

(a)

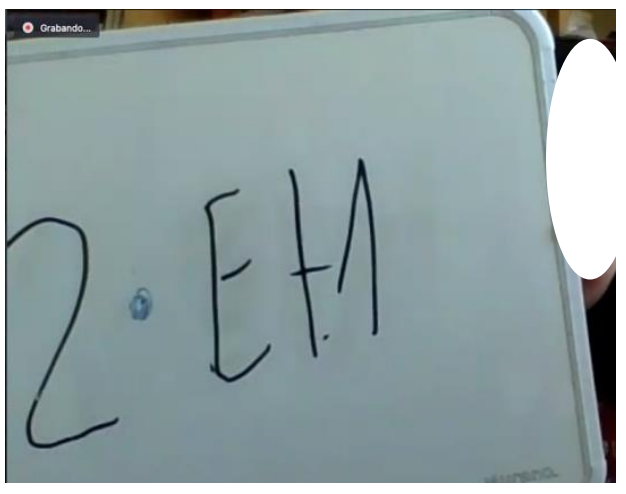

(b)

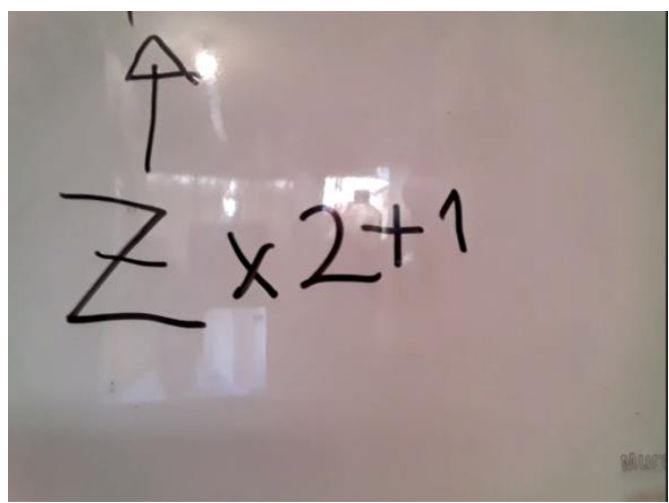

Figura 5. Respuestas escritas de dos estudiantes

Fuente: creación propia.

El pensamiento funcional ofrece la oportunidad de profundizar en la comprensión de la aritmética e interpretar las operaciones como cambio. En el caso del problema presentado, este se podría resolver como un problema con respuesta única al preguntar solo por la cantidad de personas en una parada en particular, sin embargo, al modificar los parámetros y sugerir distintos números de paradas, se ofrece la oportunidad a los estudiantes de analizar lo que permanece igual en sus cálculos y lo que cambia, reflexionar sobre los que pasa en cualquier número de paradas y 


\section{Universidade Federal da Grande Dourados}

generalizar. Incluso proponer un número tan grande como para que los alumnos no quieran o no puedan modelar el problema con cálculos puede motivarlos a buscar relaciones y propiciar el uso algebraico de los números. En Pinto et al. (2021) y Blanton et al. (2019) se describen otras situaciones problemas que permiten desarrollar el pensamiento funcional, las cuales se basan en problemas que incluyen diferentes tipos de representaciones en el enunciado.

\section{CONSIDERACIONES FINALES}

El objetivo de este trabajo era doble, por un lado, caracterizar elementos conceptuales y teóricos involucrados en el pensamiento algebraico, y por otro, reflexionar qué decisiones pedagógicas podrían fomentar el pensamiento algebraico de estudiantes de Educación Primaria. Para lograrlo, nos centramos en tres líneas de contenido al pensamiento algebraico: aritmética generalizada; equivalencia, expresiones, ecuaciones e inecuaciones; y pensamiento funcional, y en tres prácticas transversales a dichos contenidos: generalización, representación y justificación. Para conseguir este objetivo, nos apoyamos en una experiencia llevada a cabo con estudiantes de cuarto de primaria.

Uno de los puntos importantes a destacar es la posibilidad de desarrollar el pensamiento algebraico por medio del reconocimiento del carácter algebraico de la aritmética. Como se mencionó al inicio, diversas reformas curriculares han incorporado el álgebra desde los primeros cursos de primaria, por lo que es fundamental describir propuestas que permitan desarrollar el pensamiento algebraico sin incorporar nuevos contenidos, mas bien profundizar en los que ya se enseñaban. Extender las conversaciones sobre la aritmética para explorar la generalidad matemática y desarrollar habilidades algebraicas debe ser algo diario y no ocasional, o distinto a la aritmética. En este escrito mostramos alternativas en las que los estudiantes pueden desarrollar distintas habilidades a través de un problema, por ejemplo, pueden desarrollar el sentido numérico, realizar cálculos numéricos y reconocer patrones para modelar situaciones. Los problemas presentados aquí 


\section{Universidade Federal da Grande Dourados}

(sentencias numéricas, el problema de las galletas y el problema del tren) se pueden implementar en distintos cursos de primaria. La clave de lo anterior es adaptar dichos problemas a las características de los niñas y niñas, para que estas situaciones matemáticas cultiven formas de pensar cada vez más sofisticadas.

\section{AGRADECIMIENTOS}

Este artículo se enmarca en el proyecto de investigación "La construcción del pensamiento algebraico en la Formación Inicial Docente: Un estudio exploratorio" (23.400.173).

Agradecemos a los y las estudiantes que participaron de este taller, así como a los y las profesoras que nos apoyaron en el diseño e implementación de las actividades (María C. Cañadas, Marta Molina, Juan Luis Piñeiro, Marcela Carrasco, Camila Cortés, Ana María López, Valentina Ohlsen, Valentín Carril, Valentina Díaz y Rocío Moya).

\section{REFERENCIAS}

Ayala-Altamirano, C. y Molina, M. (2021). El Proceso de generalización y la generalización en acto. Un estudio de casos. PNA, 15(3), 211-241.

Blanton, M. L., Stroud, R., Stephens, A., Murphy-Gardiner, A., Stylianou, D., Knuth, E., Isler-Baylak y Strachota, S. (2019). Does early algebra matter? The effectiveness of na early álgebra intervention in grades 3 to 5 . American Educational Research Journal, 56(5), 1930-1971.

Kaput, J. J. (2008). What Is Algebra? What Is Algebraic Reasoning? En J. J. Kaput, D. W. Carraher y M. Blanton (Eds.), Algebra in the early grades (pp. 5-17). Nueva York, NY: Lawrence Erlbaum Associates. 


\section{Universidade Federal da Grande Dourados}

Kieran, C. (2004). Algebraic thinking in the early grades: What is it? The Mathematics Educator, 8(1), 139-151.

Kieran, C. (2011). Overall commentary on early algebraization: Perspectives for research and teaching. En Jinfa Cai \& E. Knuth (Eds.), Early Algebraization. A global dialogue from multiple perspectives (pp. 579-593). Países Bajos:Springer-Verlag.

Molina, M. y Castro, E. (2021). Third Grade Students' Use of Relational Thinking. Mathematics, 29(187), 1-15.

Pinto, E. y Cañadas, M. C. (2018). Generalización y razonamiento inductivo en una estudiante de cuarto de primaria. Un estudio de caso desde el pensamiento funcional. En L. J. Rodríguez-Muñiz, L. Muñiz-Rodríguez, A. AguilarGonzález, P. Alonso, F. J. García García y A. Bruno (Eds.), Investigación en Educación Matemática XXII (pp. 457- 466). Gijón: SEIEM.

Pinto, E., Cañadas, M. C y Moreno, A. (2021). Functional relationships evidenced and representations used by third graders within a functional approach to early algebra. International Journal of Science and Mathematics Education. https://doi.org/10.1007/s10763-021-10183-0

Radford, L. (2018). The Emergence of Symbolic Algebraic Thinking in Primary School. En C. Kieran (Ed.), Teaching and Learning Algebraic Thinking with 5- to 12Year-Olds (pp. 3-25). Alemania: Springer. 